\title{
EULERIAN MULTI-FLUID MODEL FOR POLYDISPERSE FLOWS
}

\section{ROBERT KESER ${ }^{1}$, ALBERTO CESCHIN ${ }^{2}$, MICHELE BATTISTONI ${ }^{3}$, HONG G. IM ${ }^{4}$ AND HRVOJE JASAK ${ }^{5}$}

${ }^{1}$ University of Zagreb, Faculty of Mechanical Engineering and Naval Architecture Ivana Lučića 5, Zagreb, Croatia robert.keser@fsb.hr

${ }^{2}$ King Abdullah University of Science and Technology, Clean Combustion Research Center Thuwal, Saudi Arabia alberto.ceschin@kaust.edu.sa

${ }^{3}$ Department of Engineering, University of Perugia Perugia, Italy michele.battistoni@unipg.it

${ }^{4}$ King Abdullah University of Science and Technology, Clean Combustion Research Center Thuwal, Saudi Arabia hong.im@kaust.edu.sa

${ }^{5}$ University of Zagreb, Faculty of Mechanical Engineering and Naval Architecture Ivana Lučića 5, Zagreb, Croatia hrvoje.jasak@fsb.hr

Key words: Eulerian multi-fluid model, Multiphase, Polydisperse, Bubbly Flow, Droplet Flow, OpenFOAM

\begin{abstract}
This work restricts the term multiphase only to disperse flows, where one of the phases is present in the form of particles, droplets or bubbles, which are suspended within the continuous phase. The dispersed elements can vary in size. The proposed method uses the classes method in the Euler-Euler framework to handle the flow's polydisperse nature. With this approach, every droplet/bubble/particle class is treated like a different phase in the calculation, i.e. every size class has its continuity and momentum equation. However, the pressure is shared among all phases. The derived model is tested for various polydisperse flows, which display the developed model's capability to predict such complex dynamic behaviour. These test cases include complex bubbly flows and dense spray (where droplet sizes vary significantly).
\end{abstract}

\section{INTRODUCTION}

Multiphase flows cover a broad range of different flow regimes. Still, this work limits its focus only to polydisperse flows, including flow topologies where at least one phase is dispersed (droplets, bubbles or particles) within the continuous phase. Furthermore, the dispersed particles are allowed to vary in their properties, e.g. diameter. 
There are three main approaches in Computational Fluid Dynamics (CFD) which offer a solution for describing such flows. The first one is the Direct Numerical Simulation (DNS) approach, which tracks and reconstructs the interface of each occurring dispersed element [1], results in substantial computational requirements. Thus, it is rarely suitable for industrial level CFD simulations.

The second approach is the Lagrangian approach [2,3], which describes the continuous phase in the Eulerian frame of reference. The dispersed elements are arranged into parcels, which are described in the Lagrangian frame of reference. The Lagrangian approach is suitable for dilute flows, where the dispersed phase volume fraction is low $[4,5]$.

The third approach is the Eulerian approach, where all phases are described in the Eulerian frame of reference. All phases are described as interpenetrating continua $[6,7,8]$, and method is suitable for a broad spectrum of various flow regimes.

This work utilizes the Eulerian approach, where the standard continuity and momentum equations are combined with the population balance equation (PBE). Furthermore, the work uses the multi-fluid formulation, which improves the model's accuracy for polydisperse flows compared to the standard two-fluid formulation. This formulation enables us to divide the dispersed elements into size classes and to treat them as different phases in the calculation.

\section{MATHEMATICAL MODEL}

This section presents the Eulerian multi-fluid model specialized for polydisperse flows and an arbitrary

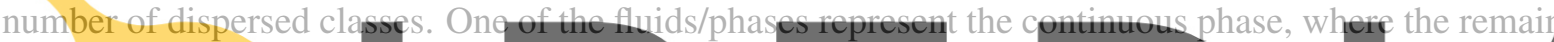
ing ones describe the disper equal diameter distributio

The employed equation $[12,13,14,15,16]$. Furt modelling. The single-phase $k-\varepsilon$
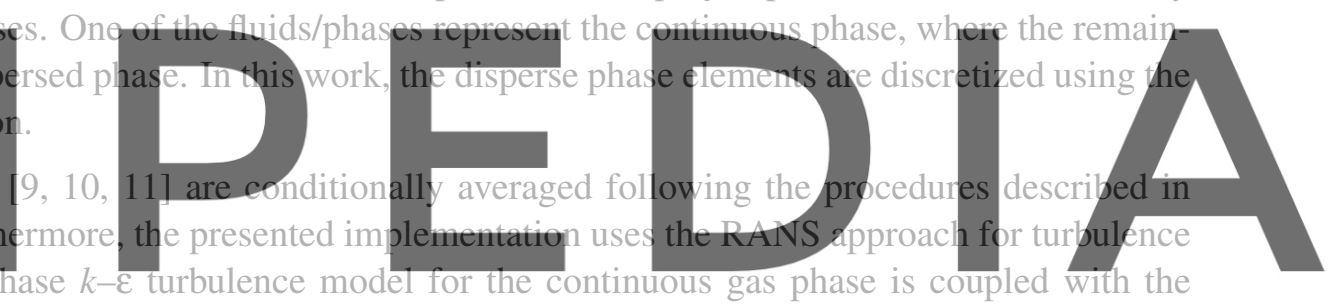

dispersed phase turbulence via the turbulence response coefficient.

Register for free at https//www.scipedia.com to download the version without the watermark The following sub-sections give the details regarding the employed momentum equations and phase fraction equations.

\subsection{Phase-intensive momentum equation}

The conditionally averaged momentum equation, which is derived in the phase-intensive form, is given by:

$$
\frac{\partial \overline{\mathbf{U}}_{\varphi}}{\partial t}+\overline{\mathbf{U}}_{\varphi} \nabla \cdot \overline{\mathbf{U}}_{\varphi}+\nabla \cdot \overline{\mathbf{R}}_{\varphi}^{\mathrm{eff}}+\frac{\nabla \alpha_{\varphi}}{\alpha_{\varphi}} \cdot \overline{\mathbf{R}}_{\varphi}^{\mathrm{eff}}=-\frac{\nabla \bar{p}}{\bar{\rho}_{\varphi}}+\mathbf{g}+\frac{\overline{\mathbf{M}}_{\varphi}}{\alpha_{\varphi} \bar{\rho}_{\varphi}}+\frac{\mathbf{S}_{\mathrm{M} \varphi}}{\alpha_{\varphi} \bar{\rho}_{\varphi}},
$$

where $\overline{\mathbf{U}}_{\varphi}$ denotes the averaged phase velocity, $\overline{\mathbf{R}}_{\varphi}^{\text {eff }}$ indicates the joined viscous and turbulent stress, $\alpha_{\varphi}$ gives the phase fraction, $\bar{p}$ is the mixture pressure, $\bar{\rho}_{\varphi}$ gives the phase density, $\mathbf{g}$ is the gravitational acceleration, $\mathbf{S}_{\mathrm{M} \varphi}$ is the net momentum source term due to breakup and coalescence processes, and $\overline{\mathbf{M}}_{\varphi}$ indicates the averaged interfacial momentum transfer term.

A detailed description and implementation of individual terms are given in $[9,12,13,14]$. 


\subsection{Phase continuity equation}

Following the derivation described by Weller [13], which was generalised for the multi-fluid formulation $[17,18]$, the modified phase continuity equation reads $[9,10,11]$ :

$$
\frac{\partial \alpha_{i}}{\partial t}+\nabla \cdot\left(\overline{\mathbf{U}} \alpha_{i}\right)+\nabla \cdot\left(\alpha_{i} \sum_{j=1, j \neq i}^{n_{\text {phases }}} \alpha_{j}\left(\overline{\mathbf{U}}_{i}-\overline{\mathbf{U}}_{j}\right)\right)=\frac{S_{i}}{\rho_{i}},
$$

where $n_{\text {phases }}$ is the total number of phases, $S_{i}$ denotes the net source term due to mass transfer between dispersed phase (bubble or droplet) classes caused by breakup and coalescence, and $\overline{\mathbf{U}}$ gives the mixture velocity, which is evaluated as:

$$
\overline{\mathbf{U}}=\sum_{i=1}^{n_{\text {phases }}} \alpha_{i} \overline{\mathbf{U}}_{i}
$$

\section{RESULTS}

This section presents the results for the previously mentioned test cases. The first sub-section deals with bubbly flows, whereas the second one gives the results obtained for droplet flow, i.e. sprays.

\subsection{Bubbly flows}

\subsubsection{Monodisperse flow}

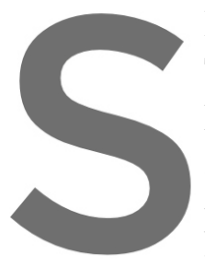

In this section, the develo

Two separate inlets with

Figure 1 gives a schemati

a diameter of 2 millimete

perimental measurements.

interfacial momentum train
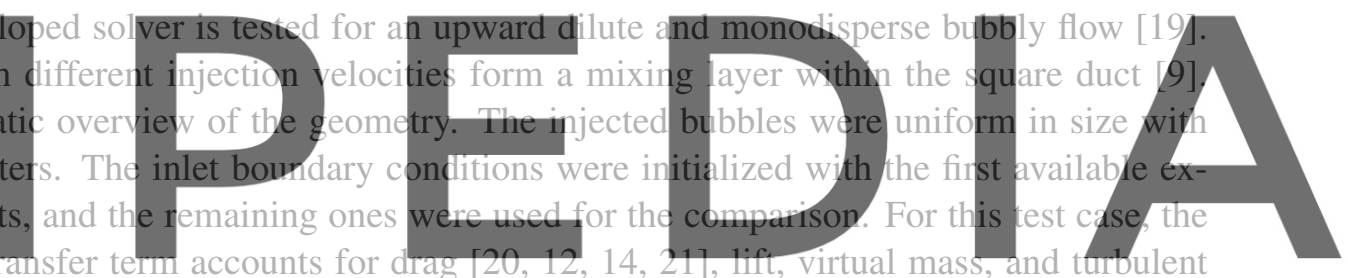

drag. This flow does not undergo breakup nor coalescence.

Register for free at https//www. scipedia.com to download the version without the watermark able.

Figure 2 shows the comparison of the calculated bubble phase fraction and the experimentally measured, and the image suggest good agreement on all sampling levels. 


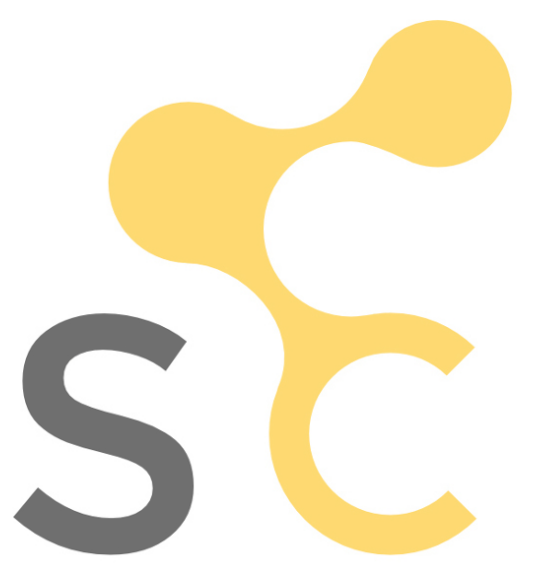

\section{Figure 1: Geometry of the square duct with two inlets.}

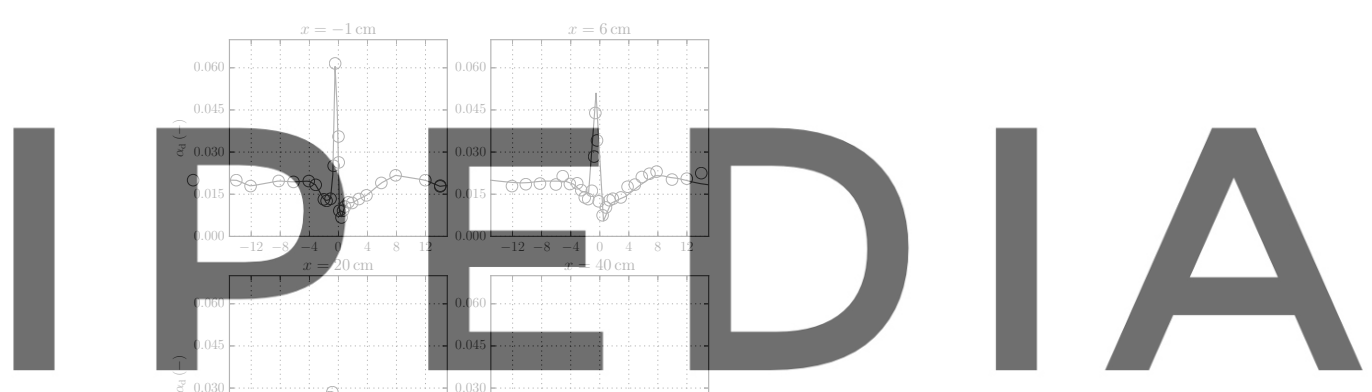

Register for free at https//www.scipedia.com to download the version without the watermark

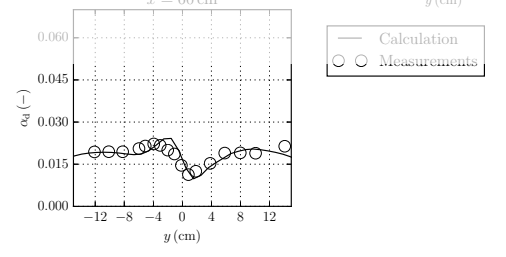

Figure 2: Comparison of the bubble phase fraction for the monodisperse test case.

\subsubsection{Polydisperse flow}

In this section, the developed solver is tested for an upward bubble flow at higher phase fractions [10]. The air was radially injected into the water stream, circulated through a large vertical pipe (the Transient twO Phase FLOW - TOPFLOW test case) [22]. For this test, we have selected the measurement point 107. Again, the first available experimental measurements were used for the initialization of the inlet boundary condition. In this case, the interfacial momentum transfer term accounts for drag (GRACE 
model [23]), lift [24, 25], virtual mass, turbulent dispersion [26], and wall lubrication [27]. Furthermore, the flow exhibits both breakup [28], and coalescence [29].

Figure 3 shows a comparison of radial bubble distribution at different sampling levels (level names were kept the same as in the experiment). The figure shows good agreement at all levels. The initial discrepancy (which is a consequence of the initialization procedure) diminishes as the bubbles travel upwards from the inlet boundary. The bubble size distribution comparison, shown in Figure 4, also suggests that the developed model can accurately reproduce the bubble behaviour for the given flow conditions.

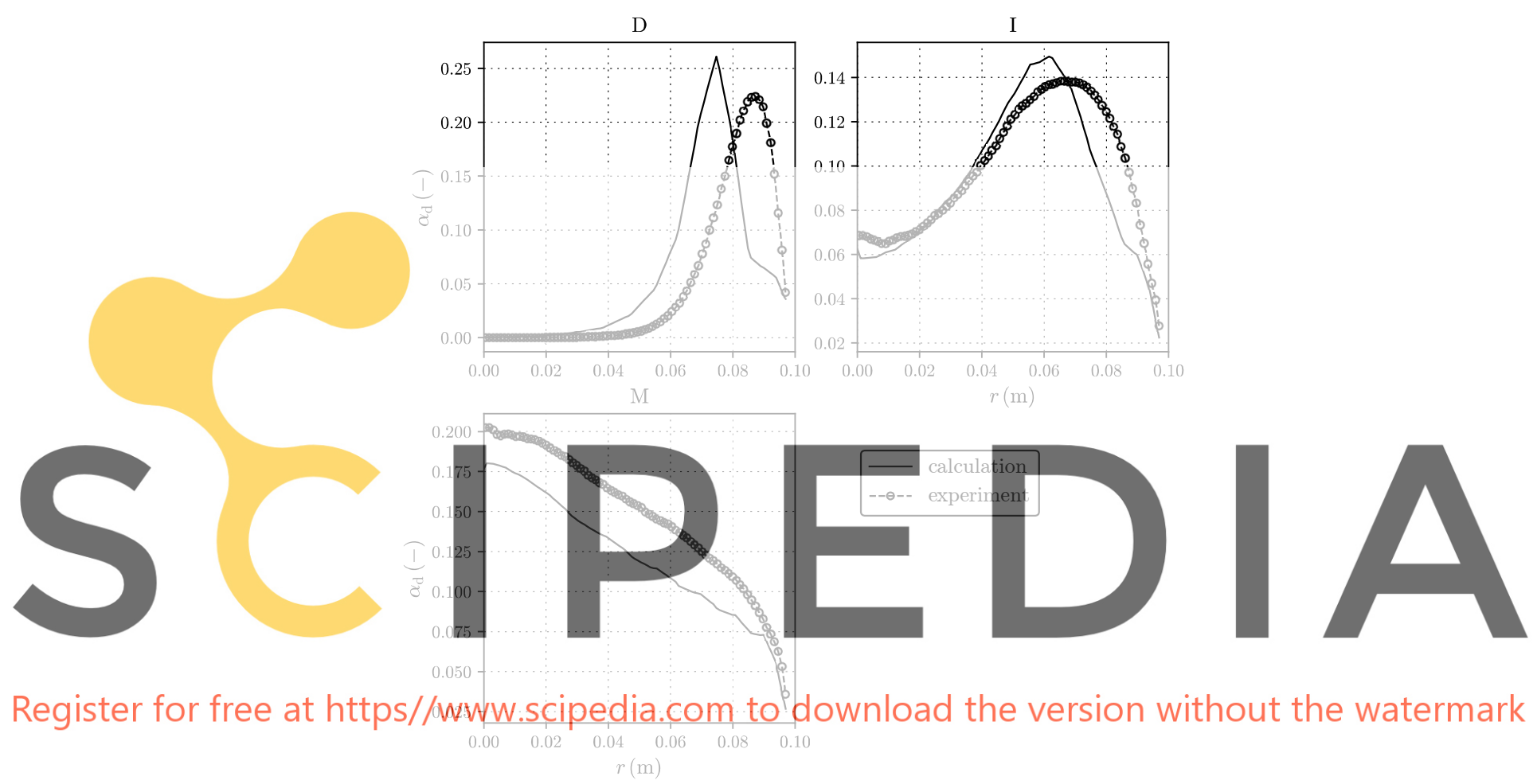

Figure 3: Comparison of the total radial bubble phase fraction profiles for the polydisperse test case. 

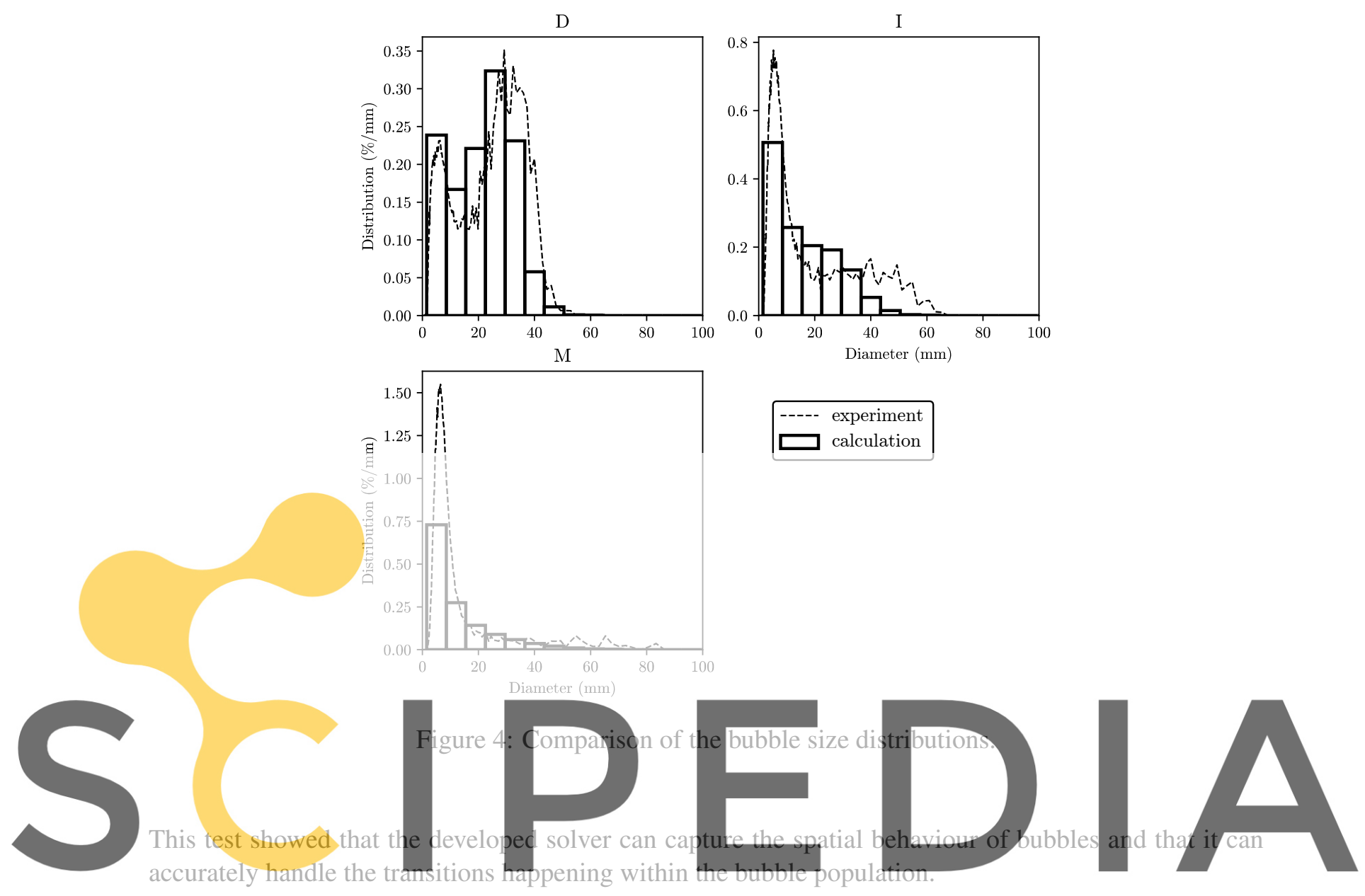

\section{Register for freeoatehttos//www.scipedia.com to download the version without the watermark}

\subsubsection{Non-evaporating spray}

In this section, the updated solver is tested for non-evaporating spray conditions. The liquid fuel. i.e. large droplets are injected through a Mini-Sac nozzle ( $140 \mu \mathrm{m}$ in diameter) into a pressurized vessel filled with carbon dioxide at 2.1 MPa. The developed solver uses a blob injection model at the inlet boundary condition, and the WAVE breakup model is responsible for the description of the breakup process. In this case, the interfacial momentum transfer term accounts for turbulent dispersion [30, 31] and drag $[32,33,34]$. Both models consider the local flow conditions, e.g. the drag model is sensitive to local droplet phase fraction, droplet deformation and Reynolds number.

Figure 6 compares the spray penetration obtained with the developed model and the experimentally measured one. The developed model adequately captures the spray behaviour, but there is a delay in the penetration in the early stage of fuel injection. The slowdown is a result of drag overprediction in the near-nozzle region. The two penetration plots are practically parallel in the remaining time frame, which implies that the spray dynamics is captured appropriately. Therefore, in future work, we intend to improve the initialization procedure, which should further enhance the accuracy in the near-nozzle 
region.

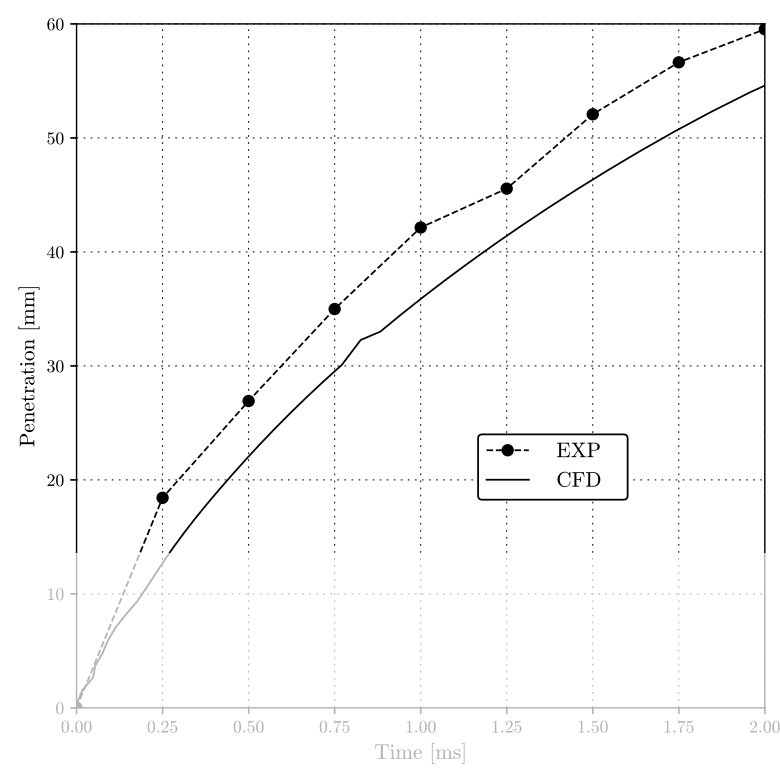

Figure 5: Spray tip penetration.

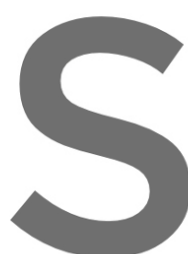

Droplet size distribution is the left sub-plot. The sub-
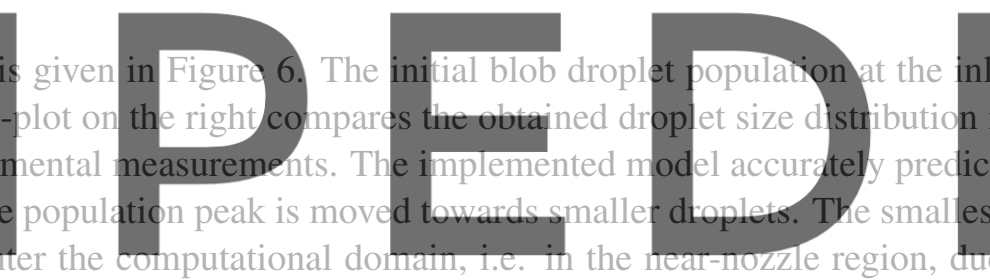

occurring droplets, but the

formed after the blobs enter

relative velocities.

Register for free at https//www.scipedia.com to download the version without the watermark
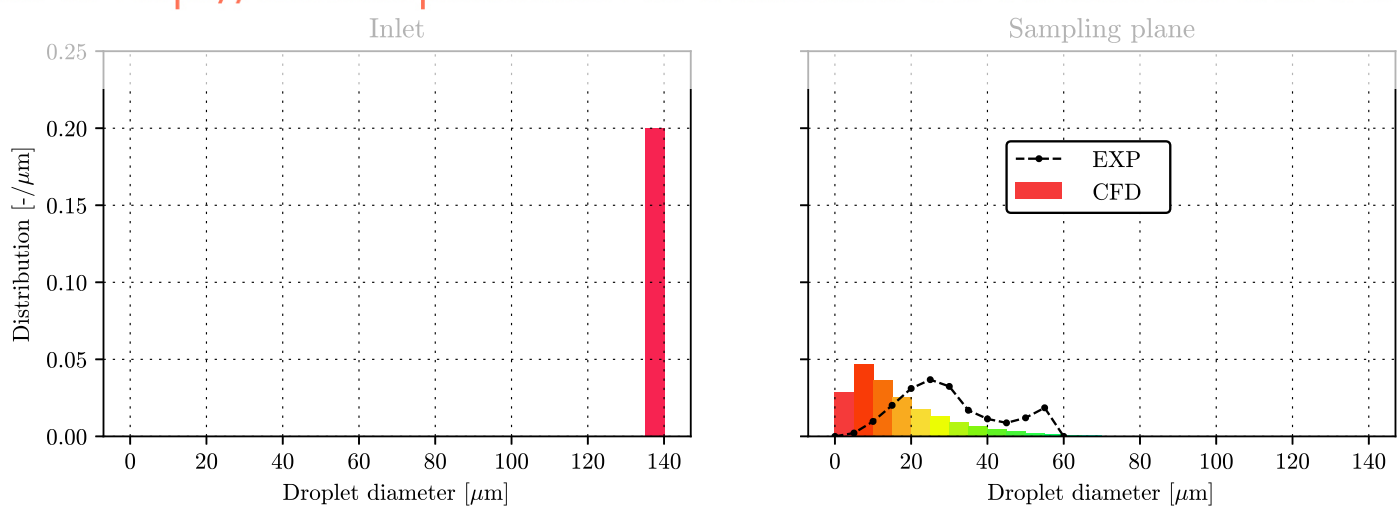

Figure 6: Droplet size distribution.

Consequently, the implemented model captures the dynamic spray adequately, but the blob injection 
approach followed by the WAVE model is not an ideal combination for describing all small-scale phenomena occurring in the near-nozzle region [11].

\subsubsection{Current developments}

We have added the energy equations for the continuous and the droplet phases to broaden the developed solver's functionality. The continuous and droplet phases are thermally coupled using the Ranz-Marshall correlation. To capture more details regarding droplets' internal thermal behaviour, e.g. finite thermal conductivity and internal recirculation within the droplets, the developed model uses a parabolic temperature profile model [35] together with the effective thermal conductivity model [36]. We are currently adding a species transfer equation which is the last prerequisite for adding single component evaporation capability.

To perform a preliminary test of the implementation, we made a simple test case. We inject droplets (with a uniform temperature of $363 \mathrm{~K}$ ) into a stationary gas at $900 \mathrm{~K}$. Also, at the inlet, we inject a small amount of gas at $900 \mathrm{~K}$. Figure 7 shows the droplet phase fraction field and the corresponding (green) sampling line in the $z$ direction. Figure 8 presents the corresponding temperatures extracted with the previously described sampling line. The passing droplets are cooling down the hot gas, and the surface temperature is slightly higher than the volume-averaged temperature.
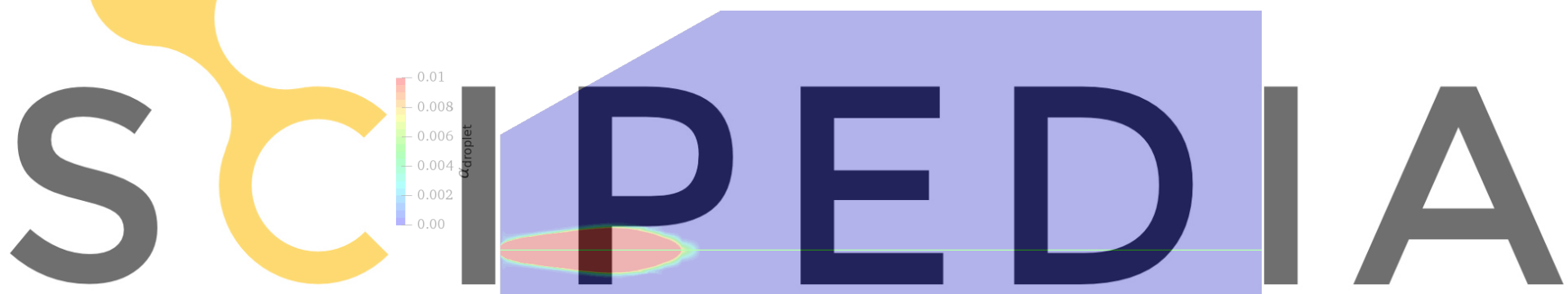

Register for free at https//www.scipedia.com to download the version without the watermark

Figure 7: Spray penetration and sampling line. 


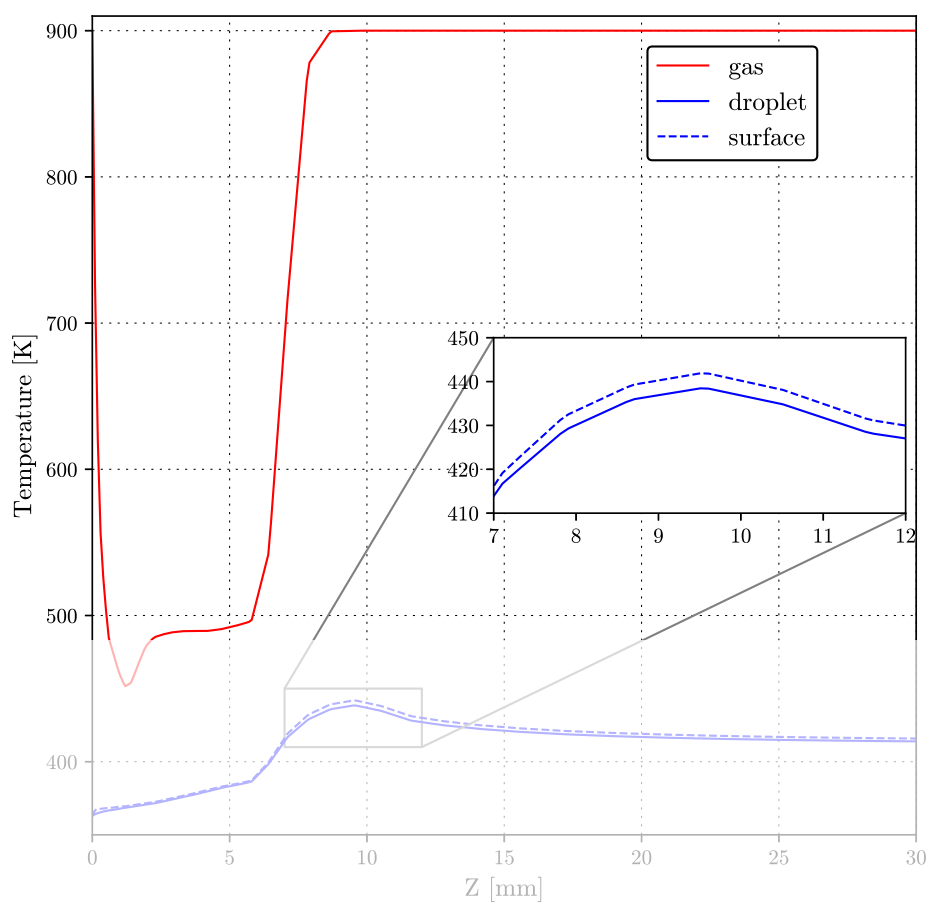

Figure 8: Temperature behaviour.

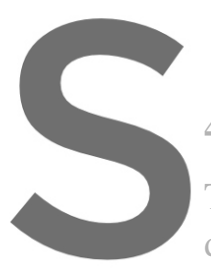

4 CONCLUSION

This work demonstrates the disperse flows using the Fuler-El
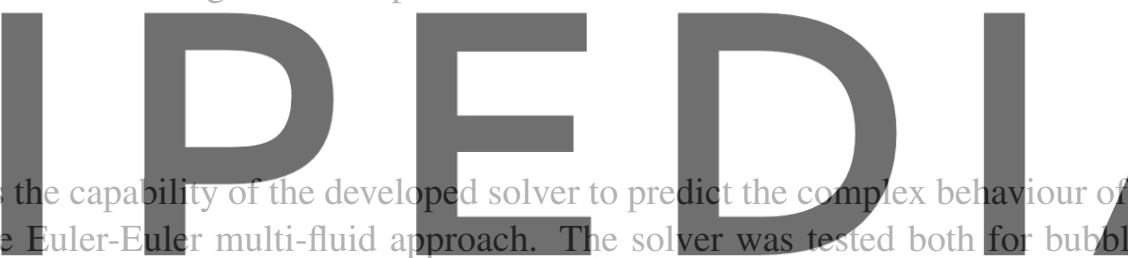

droplet flows. Overall, the solver was capable of reproducing the experimentally measured results. Fur-

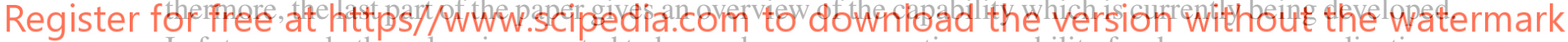 \\ In future work, the solver is expected to have advance evaporation capability for dense spray applications.}

\section{REFERENCES}

[1] Sen Han and Hanhua Zhu. Theoretical and numerical studies on the collapse of single- and doublebubble system in water. International Journal for Numerical Methods in Fluids, n/a(n/a), jul 2020.

[2] Shankar Subramaniam. Lagrangian-Eulerian methods for multiphase flows. Progress in Energy and Combustion Science, 39(2-3):215-245, apr 2013.

[3] T C Kuo, C Pan, C C Chieng, and A S Yang. EULERIAN-LAGRANGIAN COMPUTATIONS ON PHASE DISTRIBUTION OF TWO-PHASE BUBBLY FLOWS. International Journal for Numerical Methods in Fluids, 24(6):579-593, mar 1997.

[4] F. Durst, D. Miloievic, and B. Schönung. Eulerian and Lagrangian predictions of particulate twophase flows: a numerical study. Applied Mathematical Modelling, 8(2):101-115, apr 1984.

[5] Sergio Hoyas, Antonio Gil, Xandra Margot, Dung Khuong-Anh, and Frederic Ravet. Evaluation of 
the Eulerian-Lagrangian Spray Atomization (ELSA) model in spray simulations: 2D cases. Mathematical and Computer Modelling, 57(7-8):1686-1693, apr 2013.

[6] Kumar Subramanian, Johannes Zalucky, Markus Schubert, Dirk Lucas, and Uwe Hampel. An Eulerian-Eulerian Computational Approach for Simulating Descending Gas-Liquid Flows in Reactors with Solid Foam Internals. Chemical Engineering \& Technology, 40(11):2044-2057, nov 2017.

[7] Thomas Ziegenhein, Roland Rzehak, Tian Ma, and Dirk Lucas. Towards a unified approach for modelling uniform and non-uniform bubbly flows. The Canadian Journal of Chemical Engineering, 95(1):170-179, jan 2017.

[8] Mohsen Shiea, Antonio Buffo, Emilio Baglietto, Dirk Lucas, Marco Vanni, and Daniele Marchisio. Evaluation of Hydrodynamic Closures for Bubbly Regime CFD Simulations in Developing Pipe Flow. Chemical Engineering \& Technology, 42(8):1618-1626, aug 2019.

[9] Robert Keser, Vuko Vukčević, Michele Battistoni, Hong Im, and Hrvoje Jasak. Implicitly coupled phase fraction equations for the Eulerian multi-fluid model. Computers \& Fluids, 192:104277, oct 2019.

[10] Robert Keser, Alberto Ceschin, Michele Battistoni, Hong G Im, and Hrvoje Jasak. Implicitly coupled phase fraction equations for polydisperse flows. International Journal for Numerical Methods in Fluids, n/a(n/a), nov 2020.

[11] Robert Keser, Alberto Ceschin, Michele Battistoni, Hong G. Im, and Hrvoje Jasak. Development of a Eulerian Multi-Fluid Solver for Dense Spray Applications in
sep 2020.
Henrik Rusche. Computational Fluid Dynamics of Disperse
Fractions. Phd thesis, Imperial College London, 2002.

[13] Henry G Weller. Derivation modelling and solution of the conditionally averaged two-phase flow

Register for free at https//Www.Scipedia.com to down tow 2002.

[14] David Paul Hill. The Computer Simulation of Dispersed Two-phase Flows. Phd thesis, Imperial College London, 1998.

[15] Mamoru Ishii and Takashi Hibiki. Thermo-fluid dynamics of two-phase flow. 2006.

[16] J.E. Drew and S. L. Passman. Theory of Multicomponent Fluids. 1998.

[17] L. F.L.R. Silva and P. L.C. Lage. Development and implementation of a polydispersed multiphase flow model in OpenFOAM. Computers and Chemical Engineering, 35(12):2653-2666, dec 2011.

[18] Kathrin Kissling, Julia Springer, Hrvoje Jasak, Steffen Schütz, Karsten Urban, and Manfred Piesche. A coupled pressure based solution algorithm based on the volume-of-fluid approach for two or more immiscible fluids. European Conference on Computational Fluid Dynamics, (June):14-17, 2010.

[19] V. Roig, C. Suzanne, and L. Masbernat. Experimental investigation of a turbulent bubbly mixing layer. International Journal of Multiphase Flow, 24(1):35-54, feb 1998.

[20] A. Behzadi. Turbulence modelling at high phase fractions. Technical report, 2001. 
[21] William L. Haberman and R. K. Morton. An experimental investigation of the drag and shape of air bubbles rising in various liquids / by W. L. Haberman and R. K. Morton. Technical report, 2011.

[22] D. Lucas, M. Beyer, L. Szalinski, and P. Schütz. A new database on the evolution of air-water flows along a large vertical pipe. International Journal of Thermal Sciences, 49(4):664-674, apr 2010 .

[23] ANSYS CFX. ANSYS CFX-Solver Theory Guide V18.2. Technical report, 2017.

[24] Akio Tomiyama, Hidesada Tamai, Iztok Zun, and Shigeo Hosokawa. Transverse migration of single bubbles in simple shear flows. Chemical Engineering Science, 57(11):1849-1858, jun 2002.

[25] D. Lucas and A. Tomiyama. On the role of the lateral lift force in poly-dispersed bubbly flows. International Journal of Multiphase Flow, 37(9):1178-1190, nov 2011.

[26] Thomas Frank, Jun-Mei Shi, and Alan D Burns. Validation of Eulerian multiphase flow models for nuclear safety applications. In 3rd International Symposium on Two-Phase Flow Modelling and Experimentation Pisa, 22-24 September 2004, 2004.

[27] Akio Tomiyama. Struggle with computational bubble dynamics. Multiphase Science and Technology, 1998.

[28] Hean Luo and Hallvard Svendsen. A Theoretical Model for Drop or Bubble Breakup in Turbulent Dispersions, volume 42. may 1996.

[29] Michael J Prince and Harvey W Blanch. Bubble coalescence and break-up in air-sparged bubble columns. AIChE Journal, 36(10):1485-1499, oct 1990.

[30] M W Reeks. On the continuum equations for dispersed particles in nonuniform flows. Physics of Fluids A: Fluid Dynamics, 4(6):1290-1303, jun 1992.

[31] Martin A Lopez de Bertodano. Two fluid model for two-phase turbulent jets. Nuclear Engineering and Design, 179(1):65-74, 1998.

[32] Alex B Liu, Daniel Mather, and Rolf D Reitz. Modeling the Effects of Drop Drag and Breakup on Fuel Sprays. In SAE Technical Paper. SAE International, 1993.

[33] Peter J O'Rourke and Anthony A Amsden. The Tab Method for Numerical Calculation of Spray Droplet Breakup, 1987.

[34] P J O'Rourke and F V Bracco. Modeling of drop interactions in thick sprays and a comparison with experiments. Proceedings of the Institution of Mechanical Engineers, 1980.

[35] L A Dombrovsky and S. S. Sazhin. A Parabolic Temperature Profile Model for Heating of Droplets. Journal of Heat Transfer, 125(3):535-537, may 2003.

[36] William A. Sirignano. Fluid Dynamics and Transport of Droplets and Sprays. Cambridge University Press, jul 1999. 